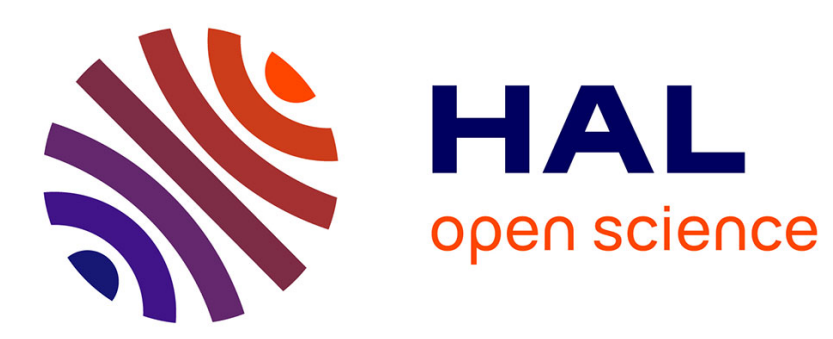

\title{
Hydrostatic interaction of a wetting fluid and a circular crack in an elastic material
}

Adélaïde Feraille Fresnet, Huy Duong Bui, Alain Ehrlacher

\section{To cite this version:}

Adélaïde Feraille Fresnet, Huy Duong Bui, Alain Ehrlacher. Hydrostatic interaction of a wetting fluid and a circular crack in an elastic material. Mechanics of Materials, 2003, 35, pp.581-586. 10.1016/S0167-6636(02)00281-8 . hal-00111372

\section{HAL Id: hal-00111372 \\ https://hal.science/hal-00111372}

Submitted on 29 Nov 2018

HAL is a multi-disciplinary open access archive for the deposit and dissemination of scientific research documents, whether they are published or not. The documents may come from teaching and research institutions in France or abroad, or from public or private research centers.
L'archive ouverte pluridisciplinaire HAL, est destinée au dépôt et à la diffusion de documents scientifiques de niveau recherche, publiés ou non, émanant des établissements d'enseignement et de recherche français ou étrangers, des laboratoires publics ou privés. 


\title{
Hydrostatic interaction of a wetting fluid and a circular crack in an elastic material
}

\author{
Adélaïde Feraille-Fresnet ${ }^{\mathrm{a}, *}$, Huy-Duong Bui ${ }^{\mathrm{b}, \mathrm{c}}$, Alain Ehrlacher ${ }^{\mathrm{a}}$ \\ ${ }^{a}$ ENPC-LAMI, 6-8 Avenue Blaise Pascal, Cité Descartes, Champs-sur-Marne, 77455 Marne-La-Vallée Cedex 2, France \\ ${ }^{\mathrm{b}}$ Laboratoire de Mécanique des Solides, Ecole Polytechnique, 91128 Palaiseau, France \\ ${ }^{\mathrm{c}}$ Division Recherche and Développement, Electricité de France, 92141 Clamart, France
}

This paper focuses on a new analytical solution: the one of the hydrostatic-elastic problem for a wetting fluid inside a 3D penny shaped circular crack in an elastic infinite solid loaded in tension at infinity, when the vapor pressure and the variation of liquid mass can be neglected. With the surface tension phenomena, the liquid is near the crack tip and is generally in tension (negative pressure).

\section{Introduction and problem position}

We are interested in different problems of fluid crack interaction. We can find such problems for example in hydraulic fracturing of rocks in the petroleum industry, or in the spalling of concrete at high temperature. The first works on the topic are related to models of $1 \mathrm{D}$ fluid flows inside the crack (Abe et al., 1976; Balueva and Zazovskii, 1985). If the crack is saturated with the fluid these 1D models give a singularity at the crack tip for the pressure field. Taking into account a twodimensional model for the fluid flow near the crack tip avoids the pressure singularity (Bui and Parnes,

\footnotetext{
${ }^{*}$ Corresponding author. Tel.: +33-1-6415-3738; fax: +33-16415-3741.

E-mail address: feraille@ceram.enpc.fr (A. Feraille-Fresnet).
}

1982), but generally authors prefer to assume that there is a lag between the fluid and the crack tips (Advani et al., 1997; Garash and Detournay, 1999; Bui, 1996). There are some physical and mechanical reasons to do such an assumption if we have a non-wetting fluid (Bui et al., 2000; Van Dam et al., 1999). On the contrary if we have a wetting liquid and if the crack does not propagate, the liquid is concentrated at the crack tip (Feraille-Fresnet and Ehrlacher, 2000). An hydrostatic-elastic solution for a non-wetting fluid inside a crack in an elastic material has been given in (Bui, 1996). In that problem, with the surface tension phenomena, the fluid is at the center of the crack, its pressure is positive and we have no fluid near the crack tip. It has shown that this can give a mechanical explanation of Rehbinder's effect (Rehbinder and Lichtman, 1957; Eecklout, 1976). More recently, Feraille-Fresnet and Ehrlacher (2000) have given the solution for a wetting liquid when there can be 
phase change between liquid and vapor. In this case, with the surface tension phenomena, the vapor is at the center of the crack at a positive pressure, and the liquid is near the crack tip. The liquid can be in tension at a negative pressure. A review of these problems can be found in FerailleFresnet et al. (2000). Taking into account the phase change is important mainly when there is an important variation of temperature. For a large family of problems, we can neglect the vapor pressure and the mass change in the liquid phase due to vaporization.

This paper deals with a new analytical solution for the hydrostatic-elastic problem of a wetting fluid inside a 3D penny shaped circular crack in an elastic infinite solid loaded in tension at infinity, when the vapor pressure and the variation of liquid mass can be neglected. With the surface tension phenomena, the liquid is near the crack tip and is generally in tension (negative pressure). For a given liquid mass inside the crack, if the loading at infinity is high enough, the crack is totally opened, but the stress intensity factor is less than it is in the case of a dried crack for the same loading. Increasing the tension at infinity involves an increase in the opening of the crack. Thus this implies an increase in the meniscus radius of the free surface of the liquid. Due to Laplace's law, the tension in the liquid decreases, which phenomenon accelerates the increase of the stress intensity factor.

For the same given liquid mass in the crack, if the tension on the solid at infinity is not high enough, the tension in the liquid near the crack tip, due to capillary phenomena, is sufficient to close the crack near the crack tip. Then the crack is partially opened and the stress intensity factor is zero.

This paper will present the non-linear relation between the loading tension at infinity and the stress intensity factor.

Consider the 3D penny shaped circular crack of radius $a$ in a linear elastic material with Lame's coefficients $\lambda$ and $\mu$. We denote $\rho$ the distance between a current point on the crack and the crack center and $r$ the dimensionless radius, such that $\rho=a r$. The incompressible fluid is wetting the solid and has a volume $V$. The fluid is near the

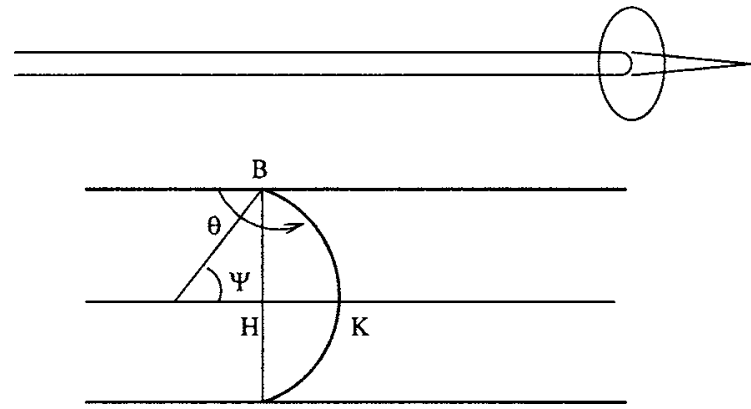

Fig. 1. Crack filled with a fluid. The lower figure represents a zoom on the circled zone of the upper one, $H B=w(c)$, $R=w(c) / \sin \psi$.

crack tip and has a uniform pressure $P$. Fig. 1 represents the crack filled with the fluid where $\theta$ is the contact angle with the solid. The angle $\theta$ is a physical characteristic of the couple fluid-solid. For example, in the case of a perfect wetting solid, $\theta=\pi$ and do not depend on the particular circulation of the contact.

The free boundary of the fluid is a curved surface which is bounded to the crack lips at a dimensionless radius $c$.

In the following analysis, we neglect the line force at the triple point (fluid, void, solid). We suppose that there is a mechanical loading at infinity in traction perpendicular to the crack surface. We denote $\sigma^{\infty}$ the uniform uniaxial stress normal to the crack surface at a long distance of the crack. Then the only loading parameters are $V$, the volume of the fluid inside the crack and $\sigma^{\infty}$. The two mains unknowns are the pressure $P$ and the dimensionless radius $c$.

\section{Problem equations}

In the frame of linear fracture mechanics, it is possible to determine the crack opening displacement of a penny shaped crack in an infinite solid as a function of $\sigma^{\infty}$ the uniform uniaxial stress at a long distance of the crack and the loading on the crack lips. Here that loading is due to the pressure $P$ of the fluid near the crack tip for dimensionless radius $r \in[c, 1]$. Then, following Bui et al. (2000), 
the crack opening displacement $w$ can be given for each dimensionless radius $r$ as a function of $P$ and c.

$$
\begin{aligned}
w(r)= & \frac{\lambda+2 \mu}{\mu(\lambda+\mu)} \frac{a}{\pi}\left(\sigma^{\infty} \sqrt{1-r^{2}}\right)+\frac{\lambda+2 \mu}{\mu(\lambda+\mu)} \\
& \times \frac{a}{\pi}\left(P \int_{\sup (c, r)}^{1} \sqrt{\frac{t^{2}-c^{2}}{t^{2}-r^{2}}} \mathrm{~d} t\right)
\end{aligned}
$$

If we neglect the volume of fluid in the meniscus, we can calculate $V(P, c)$ the volume of the fluid as a function of the two main unknowns $(P, c)$.

$$
\begin{aligned}
V(P, c) & =2 a^{2} \int_{c}^{1} 2 \pi r w(r) \mathrm{d} r \\
& =4 a^{3} \frac{\lambda+2 \mu}{\mu(\lambda+\mu)}\left[\sigma^{\infty} \frac{\left(1-c^{2}\right)^{3 / 2}}{3}+P h(c)\right]
\end{aligned}
$$

with $h(c)=\int_{c}^{1} \int_{r}^{1} r \sqrt{\left(t^{2}-c^{2}\right) /\left(t^{2}-r^{2}\right)} \mathrm{d} t \mathrm{~d} r . \quad$ As the volume of the fluid is known, we have the first relation between the two main unknowns $(P, c)$.

$V(P, c)=V$

To get a second relation we write the Laplace's law. Let us consider two fluids separated by a curved surface. We note $\chi_{1}$ and $\chi_{2}$ the two curvatures of the surface, $P^{+}$the fluid pressure on the convex side of the surface, $P^{-}$the fluid pressure on the concave side of the surface and $\tau$ the surface tension on the interface.

Laplace's law gives a necessary relation to have an equilibrium:

$P^{+}-P^{-}=\tau\left(\chi_{1}+\chi_{2}\right)$

In our situation $P^{+}$is the vapor pressure which is neglected and $P^{-}=P$. So Laplace's law can be written:

$-P=\tau\left(\chi_{1}+\chi_{2}\right)$

The geometry of the free surface of the fluid is very close to a portion of tore where the big radius is nearly $c a$ and the small radius very small in front of $c a$, as we will see after (Eq. (8)). Thus, one of the curvatures of the free surface of the fluid is well approximated by $\chi_{1}=1 / c a$.
The contact angle with the solid $\theta$, Fig. 1 , is a known characteristic of the contact between fluid and solid. We note $\psi$ the angle of the circular arc of the meniscus in the plane normal to the midplane of the crack, Fig. 1. We have a relation between $\theta, \psi$ and $\mathrm{d} w / \mathrm{d} r$ the angle of the radial tangent to the crack lips, with the radial direction in the midplane.

$\theta=-\frac{\mathrm{d} w}{\mathrm{~d} r}+\psi+\frac{\pi}{2}$

We are interested in cracks which characteristical radius has a order of magnitude of the $\mathrm{cm}$ and which opening under load before propagation of the crack is very small. Thus, in the following, we can neglect the angle $\mathrm{d} w / \mathrm{d} r$. Then $\psi$ is approximated by:

$\psi=\theta-\frac{\pi}{2}$

$\psi$ is then nearly $\pi / 2$.

Then the second curvature of the free surface of the fluid is, see Fig. 1:

$\chi_{2}=\frac{\sin \psi}{w(c)}$

where $w(c)$ is the half opening of the crack at the dimensionless radius $c$. The first curvature $\chi_{1}$ of the free surface of the fluid is negligible in front of the second curvature. Laplace's law can then be written:

$-P w(c)=\tau \sin \psi$

We have have now two expressions relating $P$ and $c$ the two unknowns of the problem. We will show in the next paragraph a way of solving it.

\section{Resolution of the problem}

It is possible to calculate $w(c)$ using (1). We denote that the integral factor of $P$ can be simply written $\int_{c}^{1} \mathrm{~d} t$. Thus:

$w(c)=\frac{\lambda+2 \mu}{\mu(\lambda+\mu)} \frac{a}{\pi}\left(\sigma^{\infty} \sqrt{1-c^{2}}+P(1-c)\right)$ 
Let us introduce $M$ :

$M=\frac{\pi}{a} \frac{\mu(\lambda+\mu)}{(\lambda+2 \mu)} \tau \sin \psi$

Introducing (10) in (9), we obtain:

$P^{2}(1-c)+P \sigma^{\infty} \sqrt{1-c^{2}}+M=0$

From (2) we get:

$\sigma^{\infty}=\frac{3 \mu(\lambda+\mu) V}{4 a^{3}(\lambda+2 \mu)\left(1-c^{2}\right)^{3 / 2}}-\frac{3 P h(c)}{\left(1-c^{2}\right)^{3 / 2}}$

Introducing (13) in (12), we obtain the following equation:

$a(c) P^{2}+b(c) P+M=0$

where

$a(c)=1-c-\frac{3 h(c)}{1-c^{2}}$

and

$b(c)=\frac{3}{4} \frac{\mu(\lambda+\mu) V}{a^{3}(\lambda+2 \mu)\left(1-c^{2}\right)}$

Eq. (14) has two solutions in $P$. From (9) we must retain the negative one:

$P(c)=\frac{-b(c)+\sqrt{b(c)^{2}-4 M a(c)}}{2 a(c)}$

Introducing (17) in (13) gives now an equation in the unknown $c$ which can be solved for each values of $V$ and $\sigma^{\infty}$.

The stress intensity factor can be written:

$K_{\mathrm{I}}(c)=2 \sqrt{\frac{a}{\pi}}\left(\sigma^{\infty}+P(c) \sqrt{1-c^{2}}\right)$

\section{Results}

We will consider that the solid is concrete and the liquid water. The following figures represent the results obtained with the following datas $a=0.1 \mathrm{~m}, \lambda=2.9 \times 10^{11} \mathrm{~Pa}, \mu=1.9 \times 10^{10} \mathrm{~Pa}$, $\tau=0.1 \mathrm{~N} \mathrm{~m}^{-1}, \psi=\pi / 2$ and $V=10^{-11} \mathrm{~m}^{3}$.

First we present the relation (17) for a dimensionless radius $c$ between 0.75 and 1, see Fig. 2. As we see, the more dried the crack (for high values of

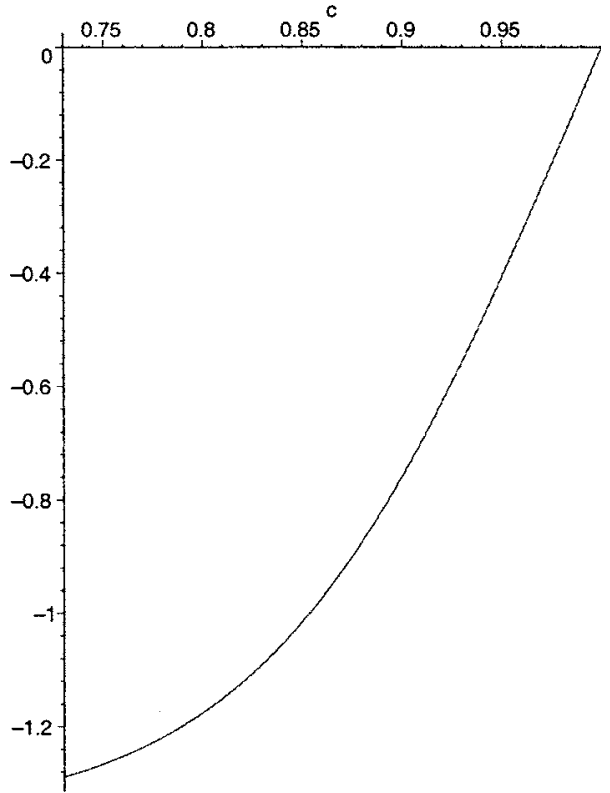

Fig. 2. Evolution of $P$ (in $\mathrm{MPa}$ ) function of $c$.

c), the lower the fluid pressure. This can be explained by the fact that, if for a given fluid volume the wetted zone is smaller, the crack is more opened and the curvature of the free boundary of the fluid is lower; then, from Laplace's law, the traction in the fluid is lower.

This point is confirmed by Fig. 3 showing the half opening of the crack as a function of $c$.

It is also confirmed in the relation giving the stress intensity factor $K_{\mathrm{I}}$ as function of $c$, see Fig. 4.

Let us have a look to the relation between the traction in the fluid function of the loading at infinity $\left(\sigma^{\infty}\right)$. Again we see that increasing loading at infinity opens the crack and then decreases the traction $(-P)$ in the fluid, see Fig. 5.

The more interesting result consists in the nonlinear relation between the stress intensity factor and the loading at infinity which can be seen on the Fig. 6. This result is more clear on the following Fig. 7 representing the ratio between the stress intensity factor of the wetted crack and the stress intensity factor that would have taken place if the crack was totally dried $\left(K_{\mathrm{I}}=2 \sqrt{a / \pi} \sigma^{\infty}\right)$. As 


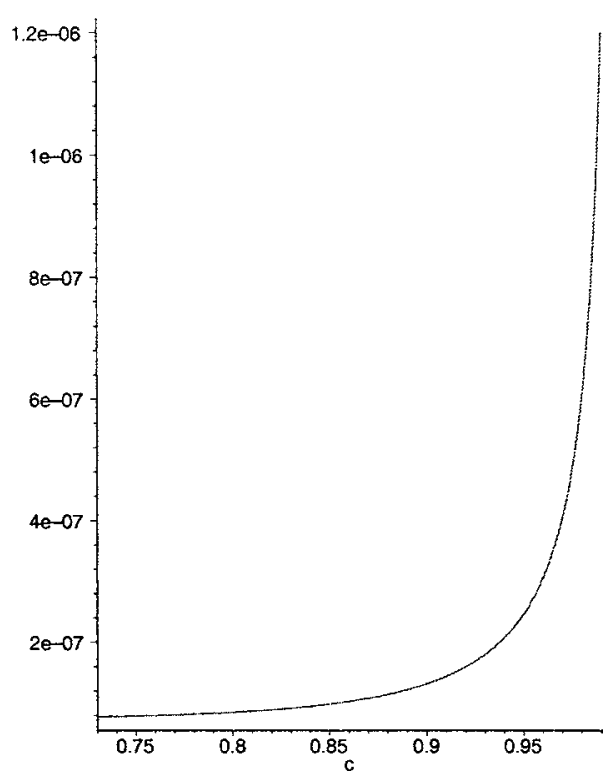

Fig. 3. Evolution of $w$ (in $\mathrm{m}$ ) function of $c$.

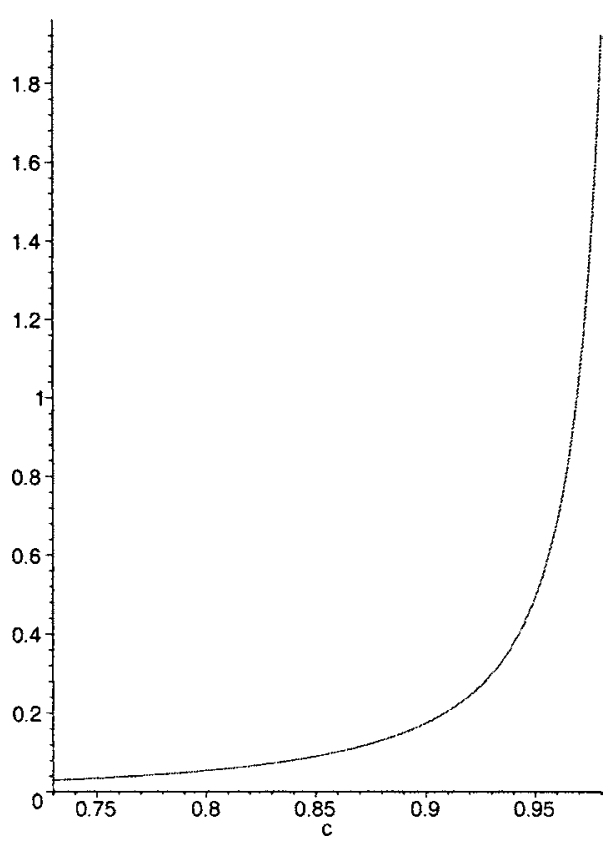

Fig. 4. Evolution of $K_{\mathrm{I}}$ (in $\mathrm{MPa} / \sqrt{m}$ ) function of $c$.

we can see the fluid in the crack decreases the stress intensity factor and so increases the apparent

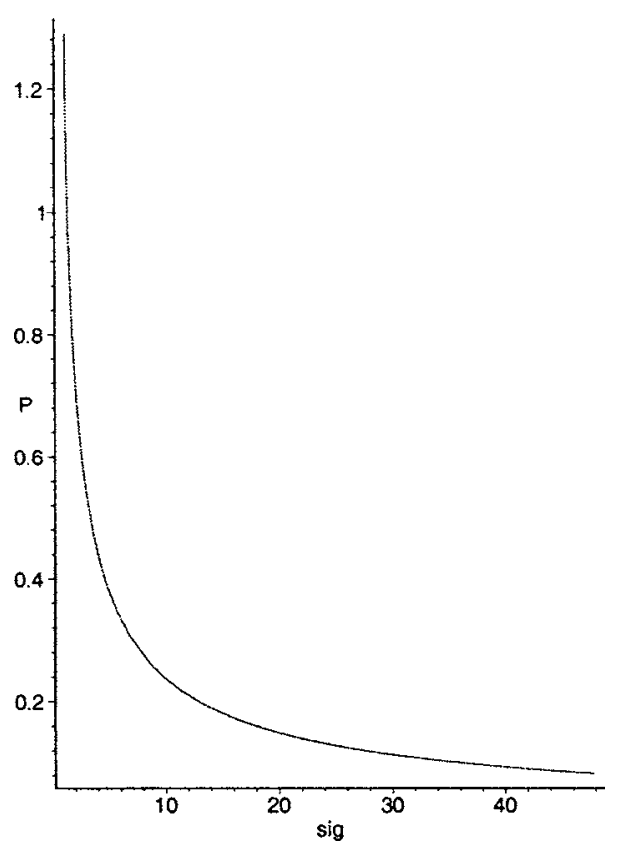

Fig. 5. Evolution of $-P$ (in $\mathrm{MPa}$ ) function of $\sigma^{\infty}$ (in $\mathrm{MPa}$ ).

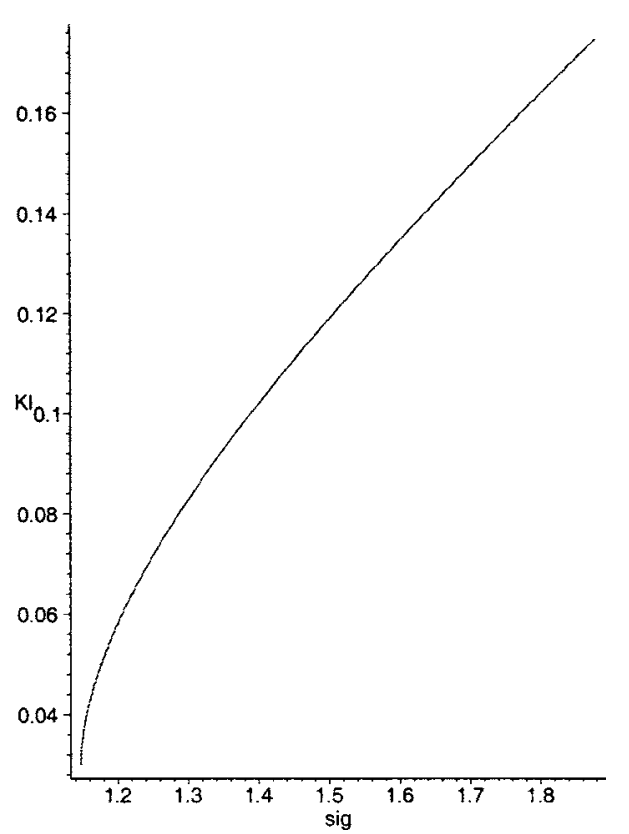

Fig. 6. Evolution of $K_{\mathrm{I}}($ in $\mathrm{MPa} / \sqrt{m})$ function of $\sigma^{\infty}$ (in MPa).

thoughtless. This phenomenon is more important if the loading at infinity is low. 


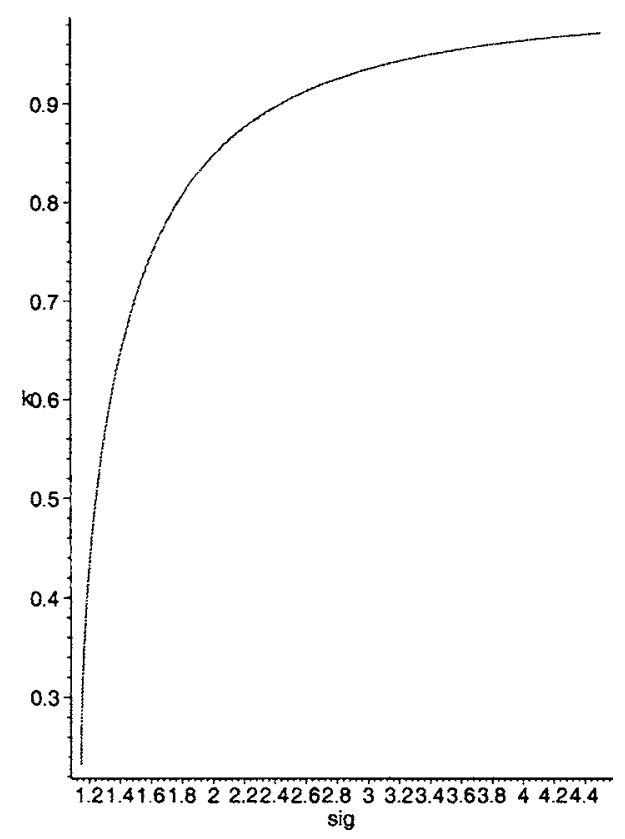

Fig. 7. Evolution of $\frac{K_{\mathrm{I}}}{2 \sqrt{a / \pi} \sigma^{\infty}}$ function of $\sigma^{\infty}$ (in $\mathrm{MPa}$ ).

\section{Conclusion}

In this paper we have presented a new analytical solution for the hydrostatic-elastic problem for a wetting fluid inside a 3D penny shaped circular crack in an elastic infinite solid loaded in tension at infinity, when the vapor pressure and the variation of liquid mass can be neglected. Due to the capillarity, the liquid is gathered at the crack tip and is generally in tension (negative pressure).

If the loading in traction at infinity is high enough, the crack is totally opened, but the stress intensity factor is less than it is in the case of a dried crack for the same loading. If we increase the loading at infinity there is an increase in the opening of the crack which implies an increase in the meniscus radius of the free surface of the liquid. Due to the Laplace's law, the tension in the liquid decreases, which phenomenon accelerates the increase of the stress intensity factor and then the strengthening due to the presence of liquid at the crack tip is getting lower.

The most interesting result of this paper is the non-linear relation between the loading tension at infinity and the stress intensity factor for a given mass of liquid due to capillarity.

\section{References}

Abe, T., Mura, T., Keer, L.M., 1976. Growth-rate of a pennyshaped crack in hydraulic fracturing of rock. J. Geophys. Res. 81, 29.

Advani, S., Lee, T., Dean, R., Pak, C., Avasthi, J., 1997. Similarity solution of a semi-infinite fluid-driven fracture in a linear elastic solid. Int. J. Numer. Anal. Meth. Geomech. 21, 229-240.

Balueva, A.V., Zazovskii, A.F., 1985. Elastic-hydrodynamic problem of inflow of fluid in a crack in porous media. Ivz. AN SSSR. Mekhanika Tverdogo Tela 20 (5), 157-166.

Bui, H.D., Parnes, R., 1982. A reexamination of the pressure at the tip of a fluid-filled crack. Int. J. Engng. Sci. 20 (11), $1215-1220$.

Bui, H.D., 1996. Interaction between the Griffith crack and a fluid: theory of Rehbin-der's effect. In: Cherepanov, G.P. (Ed.), FRACTURE: a topological encyclopedia of current knowledge. Krieger, Melbourne.

Bui, H.D., Guyon, C., Thomas, B., 2000. On viscous fluid flow near the moving crack tip. In: Maugin, G.A. et al. (Eds.), Continuum Thermomechanics: The Art and Science of Modeling Matter's Behaviour. Kluwer, Dordrecht.

Eecklout, M.V., 1976. The mechanics of strength reduction due to moisture in coal mine shales. Int. J. Rocks Mech. Min. Sci. Geomech. Abstr. 13, 61.

Feraille-Fresnet, A., Ehrlacher, A., 2000. Behaviour of a wet crack submitted to heating up to high temperature. Mech. Mater. 32, 471-484.

Feraille-Fresnet, A., Bui, H.D., Ehrlacher, A., 2000. On the role of fluids in the crack of elastic materials. In: Continuous Damage and Fracture. Elsevier, pp. 53-64.

Garash, D., Detournay, E., 1999. Similarity solution of a semiinfinite fluid-driven fracture in a linear elastic solid. Private communication.

Rehbinder, P., Lichtman, V., 1957. Effect of surface active media on strain and rupture of solid. In: Proc. 2nd Int. Cong. Surf. Act., vol. 3, pp. 563.

Van Dam, C.J., De Pater, C.J., Romijn, R., 1999. Reopening of dynamic fractures in laboratory experiments. In: Cong. Int. Mech. Rocks, Paris, II, pp. 782-794. 